\title{
Uniform Convergence of the Free Energy of the Classical Heisenberg Model to That of the Gaussian Model
}

\author{
Joseph G. Conlon ${ }^{1}$ and Jan Philip Solovej ${ }^{2}$ \\ Received April 9, 1991; final April 30, 1991
}

\begin{abstract}
We show that the free energy of the classical Heisenberg model converges to the free energy of the Gaussian in the low-temperature limit. The limit is uniform as the field is taken to zero.
\end{abstract}

KEY WORDS: Classical Heisenberg model; free energy; Gaussian model.

\section{INTRODUCTION}

We study the classical Heisenberg model defined on a $v$-dimensional cube $A=[0, L]^{v} \cap \mathbb{Z}^{v}$ by the Hamiltonian function

$$
\mathfrak{S}_{A, s}(h)=-\sum_{R \in A} \sum_{\delta \in \mathbb{Z}^{v},|\delta|=1} \bar{S}(R) \cdot \bar{S}(R+\delta)-h \sum_{R} S_{z}(R)
$$

$\mathfrak{H}_{A, s}(h)$ is a function on the space $\left(s \cdot S^{2}\right)^{\Lambda}$ of functions $\bar{S}: A \rightarrow s \cdot S^{2}$, where $s \cdot S^{2}$ denotes the sphere of radius $s>0$ centered at the origin in $\mathbb{R}^{3}$. The parameter $h>0$ represents a magnetic field in the $z$ direction. The groundstate energy, i.e., the infimum of $\mathrm{H}_{A, s}(h)$, which is attained when all spins sit at the north pole, is

$$
E_{\Lambda, s}(h)=\left(-2 v s^{2}-h s\right)|\Lambda|
$$

where $|A|$ denotes the number of sites in $A$.

\footnotetext{
${ }^{1}$ Department of Mathematics, University of Michigan, Ann Arbor, Michigan 48109.

${ }^{2}$ School of Mathematics, Institute for Advanced Study, Princeton, New Jersey 08540.
} 
We define the partition function as

$$
Z_{s}(\beta, h, \Lambda)=\int_{\left(s \cdot S^{2}\right) \cdot A \mid} \prod_{R \in A} d \bar{S}(R) \exp \left[-\beta\left(\mathfrak{H}_{A, s}(h)-E_{A, s}(h)\right)\right]
$$

Here $d \bar{S}$ is the standard Euclidean measure on $s \cdot S^{2}$ given in polar coordinates by $d \bar{S}=s^{2} \sin \varphi d \varphi d \theta$ and $\beta>0$ is the inverse temperature. Note that

$$
\mathfrak{H}_{A, s}(h)-E_{A, s}(h)=\frac{1}{2} \sum_{R \in A} \sum_{|\delta|=1}[\bar{S}(R)-\bar{S}(R+\delta)]^{2}+h \sum_{R}\left[s-S_{z}(R)\right]
$$

It is clear by a simple change of variable that

$$
Z_{s}(\beta, h, \Lambda)=s^{2|A|} Z_{s=1}\left(\beta s^{2}, h / s, \Lambda\right)
$$

We want to compare the Heisenberg partition function (1.3) with the Gaussian partition function

$$
Z_{G}(\beta, h, \Lambda)=\int_{\mathbb{C}|A|} \prod_{R \in A} d^{2} z(R) \exp \left[-\beta \mathfrak{G}_{A, G}(h)\right]
$$

where the function $\mathfrak{H}_{A, G}(h)$ defined on $\mathbb{C}^{A}$ is given by

$$
\mathfrak{S}_{\Lambda, G}(h)=\frac{1}{2} \sum_{R} \sum_{\delta}|z(R)-z(R+\delta)|^{2}+\frac{h}{2} \sum_{R}|z(R)|^{2}
$$

By identifying $\mathbb{C}$ with the tangent plane of $s \cdot S^{2}$ at the north pole, we see that

$$
\lim _{s \rightarrow \infty} Z_{s}(\beta, h, A)=Z_{G}(\beta, h, A)
$$

We are interested here in investigating this convergence in the thermodynamic limit, i.e., as $|\boldsymbol{\Lambda}| \rightarrow \infty$, and in that case study the behavior as $h$ approaches zero. Notice that the finite-volume convergence in $(1.8)$ is not uniform as $h$ tends to zero. In fact, the right-hand side diverges as $h \rightarrow 0$.

To study the thermodynamics, we compute the free energies

$$
\begin{aligned}
& f_{s}(\beta, h)=\lim _{|A| \rightarrow \infty} \frac{1}{|\Lambda|} \log Z_{s}(\beta, h, A) \\
& f_{G}(\beta, h)=\lim _{|\Lambda| \rightarrow \infty} \frac{1}{|\Lambda|} \log Z_{G}(\beta, h, A)
\end{aligned}
$$

The functions $f_{s}$ and $f_{G}$ are continuous in $\beta>0, h \geqslant 0 .{ }^{(1)}$ 
We will here prove the following results.

Theorem 1.1. For $v \geqslant 1, \beta>0$, and $h>0$ we have the limit

$$
\lim _{s \rightarrow \infty} f_{s}(\beta, h)=f_{G}(\beta, h)
$$

and the limit is uniform in $h$ as $h$ tends to zero. Thus

$$
\lim _{s \rightarrow \infty} \lim _{h \rightarrow 0} f_{s}(\beta, h)=\lim _{h \rightarrow 0} f_{G}(\beta, h)
$$

In ref. 2 we proved that (1.11) holds for $h>0$. The important result here is that the limit is uniform in $h$. The significance of the theorem is that $f_{G}(\beta, h)$ can be explicitly evaluated, and is given by

$$
f_{G}(\beta, h)=\log \frac{2 \pi}{\beta}-(2 \pi)^{-v} \int_{[-\pi, \pi]^{v}} \log [\varepsilon(k)+h] d^{\nu} k
$$

where

$$
\varepsilon(k)=4 \sum_{i=1}^{v} \sin ^{2}\left(\frac{k_{i}}{2}\right)
$$

To see this, go to Fourier variables

$$
\hat{z}(k)=|A|^{-1 / 2} \sum_{R \in A} e^{i k \cdot R} z(R)
$$

where $k$ varies in the dual lattice, i.e., $k_{i}=2 \pi r|A|^{-1 / v}, r=0,1,2, \ldots,|\Lambda|^{1 / v}-1$. Then

$$
z(R)=|\Lambda|^{-1 / 2} \sum_{k} e^{-i k \cdot R} \hat{z}(k)
$$

and

$$
\mathfrak{S}_{A, G}=\frac{1}{2} \sum_{k}[\varepsilon(k)+h]|\hat{z}(k)|^{2}
$$

We are here assuming periodic boundary conditions, i.e., we identify opposite sides of the boundary of $A$. It is well known that the free energies (1.9) and (1.10) are independent of boundary conditions.

We have now

$$
Z_{G}(\beta, h, A)=\prod_{k}\left[\frac{2 \pi}{\beta(\varepsilon(k)+h)}\right]
$$

and thus (1.13) follows upon taking the limit $|\Lambda| \rightarrow \infty$. 
From (1.5) we can state (1.12) as a low-temperature limit,

$$
\begin{aligned}
\lim _{\beta \rightarrow \infty} & \lim _{h \rightarrow 0}\left[f_{s=1}(\beta, h)+\log (\beta)\right] \\
& =\lim _{h \rightarrow 0} f_{G}(\beta=1, h) \\
& =\log (2 \pi)-(2 \pi)^{-v} \int_{[-\pi, \pi]^{v}} \log [\varepsilon(k)] d^{v}(k)
\end{aligned}
$$

This is a weak form of what we could call the classical magnon approximation. The magnon approximation is estimating the magnetization

$$
m(\beta, h)=\frac{1}{\beta}\left[\frac{\partial}{\partial h} f_{s=1}(\beta, h)\right]+1
$$

It was proved in ref. 3 that for $v \geqslant 3$ there is a phase transition in the Heisenberg model in the sense that

$$
\lim _{h \rightarrow 0} m(\beta, h)>0 \quad \text { for } \beta \text { large }
$$

This deep result was proved by using the reflection positivity property of the Hamiltonian (1.1).

On the other hand, for $v=1,2$ it is known ${ }^{(4)}$ that

$$
\lim _{h \rightarrow 0} m(\beta, h)=0 \quad \text { for all } \beta
$$

The magnon approximation, which is still an open problem, states that for $v \geqslant 3$ the magnetization can be estimated by the derivative of (1.13), i.e., we have the following:

Conjecture. For $v \geqslant 3$

$$
\lim _{\beta \rightarrow \infty} \lim _{h \rightarrow 0}[\beta(m(\beta, h)-1)]=-(2 \pi)^{-v} \int_{[-\pi, \pi]^{v}} \varepsilon(k)^{-1} d^{v} k
$$

or, stated otherwise,

$$
\lim _{h \rightarrow 0} m(\beta, h)=1-\frac{(2 \pi)^{-v}}{\beta} \int_{[-\pi, \pi]^{\prime \prime}} \varepsilon(k)^{-1} d^{v} k+o\left(\frac{1}{\beta}\right)
$$

We emphasize that our result $(1.19)$ holds for $v \geqslant 1$. Indeed, the integral in (1.19) is finite for all $v \geqslant 1$, while the integral in (1.23) is divergent for $v=1$ or 2 . 
One should observe that Theorem 1.1 implies that the expectation of the energy per unit volume at zero field will converge to its Gaussian value as $s \rightarrow \infty$. This result is not implied by the infrared bound. We have, then, the following result.

Corollary 1.2. Let $E(\beta, h)$ be the expected thermodynamic energy per unit volume,

$$
E(\beta, h)=\frac{-\partial}{\partial \beta} f_{s=1}(\beta, h)
$$

Then

$$
\lim _{\beta \rightarrow \infty} \lim _{h \rightarrow 0} \beta E(\beta, h)=1
$$

A result of the form (1.24), but for the plane rotator model (i.e., $S^{2}$ replaced by $S^{1}$ ) was proved in ref. 5 . In ref. 2 we proved an upper bound of the form (1.24).

Our proof of Theorem 1.1, which we present in the next section, is fairly elementary and does not use the infrared bound.

An interesting question is whether (1.19) holds if we replace $S^{2}$ by some other compact manifold which is, say, the boundary of a convex set in $\mathbb{R}^{3}$. For such a manifold we can of course again define a Hamiltonian similar to (1.4). It is reasonable to believe that the tangent plane approximation would still hold, at least for some points on the manifold. However, our proof relies on the special symmetry of the sphere.

\section{PROOF OF MAIN THEOREM}

As mentioned in the introduction, we proved in ref. 2 that

$$
\lim _{s \rightarrow \infty} f_{s}(\beta, h)=f_{G}(\beta, h) \quad \text { for } \quad h>0
$$

and therefore we only have to consider the uniformity as $h \rightarrow 0$. We will prove this by giving an upper and a lower bound to $f_{s}(\beta, h)$. The lower bound is easy if we observe that for $0<h \leqslant h_{0}$

$$
f_{s}(\beta, h) \geqslant f_{s}\left(\beta, h_{0}\right)
$$

This follows since the lhs of (1.4) is an increasing function of $h$. Hence from $(2.1)$

$$
\lim _{s \rightarrow \infty} \inf f_{s}(\beta, h) \geqslant f_{G}\left(\beta, h_{0}\right) \quad \text { for } \quad 0<h \leqslant h_{0}
$$

Since $f_{G}(\beta, h)$ is continuous in $h$ for $0 \leqslant h$, we conclude as follows. 
Theorem 2.1. We have

$$
\lim _{s \rightarrow \infty} \inf f_{s}(\beta, h) \geqslant f_{G}(\beta, h)
$$

uniformly in $h$ as $h$ goes to zero.

The idea in proving the upper bound is similar to the method in ref. 2 of reducing the problem to boxes of finite volume. However, it is considerably more delicate. In particular, the argument does not extend to the quantum Heisenberg ferromagnet. We first write the large box $\Lambda=[0, L]^{v} \cap \mathbb{Z}^{v}$ as a disjoint union of cubic boxes $Q_{i}$ of fixed side length $L_{0}$. We are assuming that $L / L_{0}$ is an integer. We then show that restricting the spins on the boundary $\partial Q=\bigcup_{i} \partial Q_{i}$ of all the boxes to be close to the north pole only changes the free energy by a negligible amount uniformly in $s$. This almost reduces the problem to a finite volume. We then show that one can consider all the spins as being close to the north pole, and thus approximate the sphere by its tangent plane at the north pole. That is to say that we can approximate the Heisenberg model by something smaller than the Gaussian model.

We first turn to the proof that we can restrict the spins on the boundary $\partial Q$.

Define for $A_{0} \subseteq \Lambda$ and $\Omega \subseteq S^{2}$

$$
\begin{aligned}
& Z_{s}\left(\beta, h, A, A_{0}, \Omega\right) \\
& \quad=\left(\prod_{R \in A \backslash A_{0}} \int_{s \cdot S^{2}} d \bar{S}(R)\right)\left(\prod_{R \in A_{0}} \int_{s \cdot \Omega} d \bar{S}(R)\right) \exp \left[-\beta\left(\mathfrak{S}_{\Lambda, s}-E_{A, s}\right)\right]
\end{aligned}
$$

i.e., the partition function with the spins on $A_{0}$ restricted to $s \cdot \Omega$. Let $\Omega\left(\varphi_{0}\right) \subseteq S^{2}$ be the solid angle around the north pole given in polar coordinates by $\left\{(\theta, \varphi) \mid 0 \leqslant \theta \leqslant 2 \pi, 0 \leqslant \varphi \leqslant \varphi_{0}\right\}$.

Lemma 2.2. If $\left|\Omega\left(\varphi_{0}\right)\right|$ denotes the area of $\Omega\left(\varphi_{0}\right)$, we have

$$
Z_{s}(\beta, h, \Lambda) \leqslant\left(\frac{c}{\left|\Omega\left(\varphi_{0}\right)\right|}\right)^{\left|A_{0}\right|} Z_{s}\left(\beta, h, A, \Lambda_{0}, \Omega\left(\varphi_{0}\right)\right)
$$

for a universal constant $c$.

It is in the proof of this lemma that we will rely on the symmetry of the sphere. The estimate (2.6) will be an immediate consequence of the following two lemmas. 
Lemma 2.3. Let $\bar{S}_{1}, \ldots, \bar{S}_{n} \in S^{2}$. Then

$$
\int_{S^{2}} \prod_{i=1}^{n}\left(\bar{S}_{i} \cdot \bar{S}\right) d \bar{S}=c_{n} \sum_{D} \prod_{(i, j) \in D}\left(\bar{S}_{i} \cdot \bar{S}_{j}\right)
$$

where the sum is over pair decompositions

$$
D=\left\{\left(i_{1}, i_{2}\right), \ldots,\left(i_{n-1}, i_{n}\right)\right\}, \quad \text { of } \quad\{1, \ldots, n\}=\left\{i_{1}, \ldots, i_{n}\right\}, \quad c_{n}>0
$$

if $n$ is even, $c_{n}=0$ if $n$ is odd.

Proof. Consider the Gaussian integral

$$
\int_{\mathrm{R}^{3}} \exp \left[\sum_{i=1}^{n} \lambda_{i}\left(\bar{S}_{i} \cdot x\right)-x^{2}\right] d x=F\left(\lambda_{1}, \ldots, \lambda_{n}\right)
$$

Then we have

$$
\begin{array}{r}
\left.\frac{\partial}{\partial \lambda_{1}} \cdots \cdot \frac{\partial}{\partial \lambda_{n}} F\left(\lambda_{1}, \ldots, \lambda_{n}\right)\right|_{\lambda_{1}=\lambda_{2}=\ldots=\lambda_{n}=0} \\
\quad=\int_{S^{2}} \prod_{i=1}^{n}\left(\bar{S}_{i} \cdot \bar{S}\right) d \bar{S} \int_{0}^{\infty} r^{n+2} e^{-r^{2}} d r
\end{array}
$$

On the other hand, we can evaluate the Gaussian integral explicitly,

$$
F\left(\lambda_{1}, \ldots, \lambda_{n}\right)=\pi^{3 / 2} \exp \left[\frac{1}{4}\left(\sum_{i=1}^{n} \lambda_{i} \bar{S}_{i}\right)^{2}\right]
$$

Performing the differentiation on $F$, we obtain the result.

The second result we need to prove (2.6) is the following estimate, which should be intuitively clear.

Lemma 2.4. Let $r_{i j}, r_{i}, 1 \leqslant i, j \leqslant n$, be nonnegative integers. Then for $0 \leqslant \varphi_{0} \leqslant \pi / 2$

$$
\begin{aligned}
& \int_{S^{2}} d \bar{S}_{1} \cdots \int_{S^{2}} d \bar{S}_{n} \prod_{i, j=1}^{n}\left|\left(\bar{S}_{i} \cdot \bar{S}_{j}\right)\right|^{r_{i j}} \prod_{i=1}^{n}\left|\left(\bar{S}_{i} \cdot \bar{k}\right)\right|^{r_{i}} \\
& \quad \leqslant\left(\frac{\pi^{4}}{\left|\Omega\left(\varphi_{0}\right)\right|}\right)^{n} \int_{\Omega\left(\varphi_{0}\right)} d \bar{S}_{1} \cdots \cdot \int_{\Omega\left(\varphi_{0}\right)} d \bar{S}_{n} \prod_{i, j=1}^{n}\left|\left(\bar{S}_{i} \cdot \bar{S}_{j}\right)\right|^{r_{i j}} \prod_{i=1}^{n}\left|\left(\bar{S}_{i} \cdot \bar{k}\right)\right|^{r_{i}}
\end{aligned}
$$

where $\bar{k}$ is the unit vector in the direction of the $z$ axis. 
Proof. Denote the integrand in (2.11) by $F\left(\bar{S}_{1}, \ldots, \bar{S}_{n}\right)$. If $\Omega_{+}$denotes the part of $S^{2}$ in the first octant, i.e., $\Omega_{+}=\{(\theta, \varphi) \mid 0 \leqslant \theta \leqslant \pi / 2$, $0 \leqslant \varphi \leqslant \pi / 2\}$, then from the inequality

$$
\left|\bar{S}_{i} \cdot \bar{S}_{j}\right| \leqslant\left|S_{i}^{x} S_{j}^{x}\right|+\left|S_{i}^{y} S_{j}^{y}\right|+\left|S_{i}^{z} S_{j}^{z}\right|
$$

we get

$$
\int_{S^{2}} d \bar{S}_{1} \cdots \int_{S^{2}} d \bar{S}_{n} F \leqslant 8^{n} \int_{\Omega_{+}} d \bar{S}_{1} \cdots \int_{\Omega_{+}} d \bar{S}_{n} F
$$

Now let, for $\lambda \geqslant 1, \tau_{\lambda}: \Omega_{+} \rightarrow \Omega_{+}$be defined by $\tau_{\lambda}(\theta, \varphi)=(\theta, \varphi / \lambda)$. Then, as can be easily checked by differentiation,

$$
F\left(\tau_{\lambda}\left(\bar{S}_{1}\right), \ldots, \tau_{\lambda}\left(\bar{S}_{n}\right)\right) \geqslant F\left(\bar{S}_{1}, \ldots, \bar{S}_{n}\right)
$$

for $\bar{S}_{1}, \ldots, \bar{S}_{n} \in \Omega_{+}$. Thus,

$$
\begin{aligned}
\int_{\Omega_{+}} d \bar{S}_{1} & \ldots \cdot \int_{\Omega_{+}} d \bar{S}_{n} F\left(\bar{S}_{1}, \ldots, \bar{S}_{n}\right) \\
= & \int_{\tau_{\lambda}\left(\Omega_{+}\right)} d \theta_{1} d \varphi_{1} \lambda \sin \left(\lambda \varphi_{1}\right) \\
& \cdots \cdot \int_{\tau_{i}\left(\Omega_{+}\right)} d \theta_{n} d \varphi_{n} \lambda \sin \left(\lambda \varphi_{n}\right) F\left(\tau_{\lambda}^{-1}\left(\bar{S}_{1}\right), \ldots, \tau_{\lambda}^{-1}\left(\bar{S}_{n}\right)\right) \\
\leqslant & {\left[\frac{\pi \lambda^{2}}{2}\right]^{n} \int_{\tau_{\lambda}\left(\Omega_{+}\right)} d \bar{S}_{1} \ldots \int_{\tau_{\lambda}\left(\Omega_{+}\right)} d \bar{S}_{n} F\left(\bar{S}_{1}, \ldots, \bar{S}_{n}\right) }
\end{aligned}
$$

where we have used (2.13) and that $\sin (\lambda \varphi) \leqslant(\pi / 2) \lambda \sin \varphi$ for $\lambda \geqslant 1$ and $0 \leqslant \lambda \varphi \leqslant \pi / 2$. If we choose $\lambda=\pi / 2 \varphi_{0}$ and use $\left|\Omega\left(\varphi_{0}\right)\right|=$ $2 \pi\left(1-\cos \varphi_{0}\right) \leqslant \pi \phi_{0}^{2}$, (2.11) follows from (2.14).

Proof of Lemma 2.2. We can of course assume that $\varphi_{0}$ is so small that the inner product of two vectors in $\Omega\left(\varphi_{0}\right)$ is positive. To prove (2.6), expand the exponential $\exp \left[-\beta \mathfrak{G}_{A, s}(h)\right]$ appearing in the integrand of $Z_{s}$. In each single term do the integral over the variables $\bar{S}(R)$ for $R \in A \backslash \Lambda_{0}$. From Lemma 2.3 the remaining terms that all appear with positive coefficients are of the form in (2.11) without the absolute values. Hence (2.6) follows from Lemma 2.4 .

We are now in a position to prove that we can restrict all the spins to be close to the north pole. 
Lemma 2.5. Given $\varepsilon>0$, and let $0 \leqslant \varphi_{0} \leqslant \pi / 4$; then there exists $s_{0}>0$ depending only on $\varepsilon, \beta, L_{0}$, and $\varphi_{0}$, but not on $h$, such that if $s \geqslant s_{0}$, then

$$
Z_{s}\left(\beta, h, A, \partial Q, \Omega\left(\varphi_{0}\right)\right) \leqslant \exp [\varepsilon|A|] Z_{s}\left(\beta(1-\varepsilon), h, A, A, \Omega\left(2 \varphi_{0}\right)\right)
$$

Proof. We denote by $Z\left(Q_{1}, \ldots, Q_{n}\right)$ the partition function with variables on $\partial Q$ restricted to $s \cdot \Omega\left(\varphi_{0}\right)$ and at least one variable in each box $Q_{1}, \ldots, Q_{n}$ restricted outside $s \cdot \Omega\left(2 \varphi_{0}\right)$, i.e., in $s \cdot S^{2} \backslash s \cdot \Omega\left(2 \varphi_{0}\right)$, and all the remaining variables, on $A \backslash\left(\bigcup_{i=1}^{n} Q_{i} \cup \partial Q\right)$ restricted to $s \cdot \Omega\left(2 \varphi_{0}\right)$. Then we have

$$
\begin{aligned}
& Z_{s}\left(\beta, h, \Lambda, \partial Q, \Omega\left(\varphi_{0}\right)\right) \\
& \quad \leqslant Z_{s}\left(\beta, h, A, A, \Omega\left(2 \varphi_{0}\right)\right)+\sum_{i} Z\left(Q_{i}\right)+\sum_{i<j} Z\left(Q_{i}, Q_{j}\right)+\cdots
\end{aligned}
$$

It is clear that for the configurations integrated over in order to compute $Z\left(Q_{i}\right)$ we have

$$
\mathfrak{S}_{\Lambda}-E_{A} \geqslant \frac{1}{2} \sum_{R, \delta}[\bar{S}(R+\delta)-\bar{S}(R)]^{2} \geqslant C\left(L_{0}, \varphi_{0}\right) s^{2}
$$

where $C\left(L_{0}, \varphi_{0}\right)>0$. Thus we have

$$
\begin{aligned}
Z\left(Q_{i}\right) & \leqslant \exp \left[-\varepsilon \beta C\left(L_{0}, \varphi_{0}\right) s^{2}\right] Z_{s}\left(\beta(1-\varepsilon), h, A, A \backslash Q_{i}, \Omega\left(2 \varphi_{0}\right)\right) \\
& \leqslant \exp \left[-\varepsilon \beta C\left(L_{0}, \varphi_{0}\right) s^{2}\right]\left[\frac{c}{\left|\Omega\left(2 \varphi_{0}\right)\right|}\right]^{L_{0}^{\prime}} Z_{s}\left(\beta(1-\varepsilon), h, A, A, \Omega\left(2 \varphi_{0}\right)\right)
\end{aligned}
$$

where in the last estimate we have used an obvious generalization of Lemma 2.2. We can now clearly choose $s_{0}$ such that for $s \geqslant s_{0}$

$$
Z\left(Q_{i}\right) \leqslant \varepsilon Z_{s}\left(\beta(1-\varepsilon), h, A, A, \Omega\left(2 \varphi_{0}\right)\right)
$$

Similarly, we get for the same choice of $s_{0}$

$$
Z\left(Q_{1}, \ldots, Q_{n}\right) \leqslant \varepsilon^{n} Z_{s}\left((1-\varepsilon) \beta, h, A, A, \Omega\left(2 \varphi_{0}\right)\right)
$$

Thus, from (2.16),

$$
Z_{s}\left(\beta, h, A, \partial Q, \Omega\left(\varphi_{0}\right)\right) \leqslant(1+\varepsilon)^{|A| / \mathcal{L}_{0}^{v}} Z_{s}\left((1-\varepsilon) \beta, h, A, A, \Omega\left(2 \varphi_{0}\right)\right)
$$

from which (2.15) immediately follows. 
Finally we can now prove the upper bound on the free energy.

Theorem 2.6. For any $\delta>0$ there exists $s_{0}>0$ depending on $\delta$ such that that if $s \geqslant s_{0}$

$$
f_{s}(\beta, h) \leqslant f_{G}(\beta(1-\delta), h)+\delta
$$

Proof. From Lemmas 2.2 and 2.5 we can, for all $\varepsilon, \beta, L_{0}$, and $0 \leqslant \varphi_{0} \leqslant \pi / 4$, find $s_{0}$ such that for $s \geqslant s_{0}$

$Z_{s}(\beta, h, \Lambda) \leqslant\left[\frac{c}{\left|\Omega\left(\varphi_{0}\right)\right|}\right]^{|\partial Q|} \exp [\varepsilon|\Lambda|] Z_{s}\left(\beta(1-\varepsilon), h, A, A, \Omega\left(2 \varphi_{0}\right)\right)$

By choosing $\varphi_{0}$ small enough and comparing the sphere by, say, stereographic projection with the tangent plane at the north pole, we easily get

$$
Z_{s}\left(\beta(1-\delta / 3), h, \Lambda, \Lambda, \Omega\left(2 \varphi_{0}\right)\right) \leqslant \exp \left[\frac{\delta}{3}|\Lambda|\right] Z_{G}\left(\beta(1-\delta / 3)^{2}, h, \Lambda\right)
$$

Now choose $L_{\ddot{0}}$ so large that (we can assume $|\Lambda|$ as large as we please)

$$
\left[\frac{c}{\left|\Omega\left(\varphi_{0}\right)\right|}\right]^{|\partial \Omega|} \leqslant\left[\frac{c}{\left|\Omega\left(\varphi_{0}\right)\right|}\right]^{|A| / L_{0}^{\nu} \cdot 2 v L_{0}^{\nu-1}}<\exp \left[\frac{\delta}{3}|A|\right]
$$

For this $L_{0}$, the above chosen $\varphi_{0}$, and $\varepsilon=\delta / 3$, choose $s_{0}$ such that (2.23) holds for $s \geqslant s_{0}$. Then from (2.24) we have for $s \geqslant s_{0}$

$$
Z_{s}(\beta, h, \Lambda) \leqslant \exp [\delta|\Lambda|] Z_{G}\left(\beta(1-\delta / 3)^{2}, h,|\Lambda|\right)
$$

Taking the thermodynamic limits on both sides gives (2.22).

Proof of Theorem 1.1. We only have to argue that

$$
\limsup _{s \rightarrow \infty} f_{s}(\beta, h) \leqslant f_{G}(\beta, h)
$$

uniformly as $h$ goes to zero. This immediately follows from (2.22) since $f_{G}(\beta, h)$ is equicontinuous in $h$ as $h$ goes to zero.

Proof of Corollary 1.2. We use (1.19) and the fact that $f_{s=1}(\beta, h)$ is a convex function of $\beta$. Hence, for any $\varepsilon>0$ we have

$$
\frac{f_{s=1}(\beta, h)-f_{s=1}(\beta(1+\varepsilon), h)}{\varepsilon}<\beta E(\beta, h)<\frac{f_{s=1}(\beta(1-\varepsilon), h)-f_{s=1}(\beta, h)}{\varepsilon}
$$


Now let us put

$$
f_{s=1}(\beta, h)=-\log (\beta)+c(\beta, h)
$$

and

$$
\lim _{h \rightarrow 0} c(\beta, h)=c(\beta)
$$

Then (1.19) implies that the limit

$$
\lim _{\beta \rightarrow \infty} c(\beta)=c(\infty) \text { exists }
$$

From (2.28) we have

$$
\begin{aligned}
& {[\log (1+\varepsilon)+c(\beta)-c(\beta(1-\varepsilon))] / \varepsilon} \\
& \quad<\lim _{h \rightarrow 0} \beta E(\beta, h) \\
& \quad<[-\log (1-\varepsilon)+c(\beta(1-\varepsilon))-c(\beta)] / \varepsilon
\end{aligned}
$$

Hence from (2.31) we have

$$
\frac{\log (1+\varepsilon)}{\varepsilon}<\lim _{\beta \rightarrow \infty} \lim _{h \rightarrow 0} \beta E(\beta, h)<\frac{-\log (1-\varepsilon)}{\varepsilon}
$$

Taking $\varepsilon \rightarrow 0$ now yields (1.26).

\section{ACKNOWLEDGMENTS}

This work was partially supported by U.S. NSF grants DMS 8900244 (J.G.C.) and DMS 9002416 (J.P.S.). The authors would like to thank Michael Aizenman and Paul Federbrush for helpful conversations.

\section{REFERENCES}

1. [R] D. Ruelle, Statistical Mechanics (Benjamin, New York, 1969).

2. [CS] J. G. Conlon and J. P. Solovej, On asymptotic limits for the quantum Heisenberg model, J. Phys. A Math. Gen. 23:3199-3213 (1990).

3. [FSS] J. Fröhlich, B. Simon, and T. Spencer, Infrared bounds, phase transitions and continuous symmetry breaking, Commun. Math. Phys. 50:79-85 (1976).

4. N. Mermin, Absence of ordering in certain classical systems, J. Math. Phys. 8:1061-1064 (1967).

5. [BFLLS] J. Bricmont, J.-R. Fontaine, J. L. Lebowitz, E. H. Lieb, and T. Spencer, Lattice systems with a continuous symmetry III, Commun. Math. Phys. 78:545-566 (1981). 